\title{
Inflammatory bowel disease and risk of urinary cancers: a systematic review and pooled analysis of population-based studies
}

\author{
Dechao Feng ${ }^{1,2 \#}$, Yubo Yang ${ }^{1 \#} \wedge$, Zhenghao Wang ${ }^{1}$, Wuran Wei ${ }^{1} \wedge, \mathrm{Li} \mathrm{Li}^{2,3}$ \\ ${ }^{1}$ Department of Urology, Institute of Urology, West China Hospital, Sichuan University, Chengdu, China; ${ }^{2}$ Key Laboratory of Birth Defects and \\ Related Diseases of Women and Children (Sichuan University), Ministry of Education, Chengdu, China; ${ }^{3}$ Department of Pediatrics, West China \\ Second University Hospital, Sichuan University, Chengdu, China \\ Contributions: (I) Conception and design: D Feng; (II) Administrative support: L Li, W Wei; (III) Provision of study materials or patients: D Feng, \\ Y Yang; (IV) Collection and assembly of data: Y Yang, Z Wang; (V) Data analysis and interpretation: D Feng, Y Yang; (VI) Manuscript writing: All \\ authors; (VII) Final approval of manuscript: All authors. \\ "These authors contributed equally to this work. \\ Correspondence to: Li Li. Department of Pediatrics, West China Second University Hospital, Sichuan University, No. 20, Section 3, Renmin Nan Lu, \\ Chengdu 610041, China. Email: liliwch@126.com.
}

Background: The aim of this study is to elucidate the risk of urologic cancers in patients with Crohn's disease (CD) and ulcerative colitis (UC).

Methods: Electronic databases including PubMed, the Cochrane Library, Embase and Web of Science, and manual retrieval were conducted from inception to June 2020. Two reviewers independently searched the above databases and selected the studies using prespecified standardized criteria. The Newcastle-Ottawa Scale was used to assess the risk of bias in the included studies, and this meta-analysis was completed by STATA version 14.2.

Results: A total of 12 cohort studies and 4 case-control studies were included in this meta-analysis. Overall, patients with inflammatory bowel disease (IBD) were at significantly increased risk of renal cancer (RCa) [standardized incidence ratio (SIR): $1.53 ; 95 \%$ confidence interval (CI): $1.25-1.80 ; \mathrm{I}^{2}=42.4 \%$, but not at increased risk of prostate cancer $(\mathrm{PCa})$, bladder cancer $(\mathrm{BCa})$ and male genital cancer. In the subgroup analysis, CD patients had a significantly higher RCa risk (SIR: 1.95; 95\% CI: $1.45-2.44 ; \mathrm{I}^{2}=39.9 \%$ ). Besides, CD patients seemed to be at borderline significantly increased risks of PCa (SIR: 1.07; 95\% CI: 0.93-1.20; $\mathrm{I}^{2}=15.1 \%$ ) and BCa (SIR:1.19; 95\% CI: 0.94-1.44; $\mathrm{I}^{2}=0 \%$ ), and UC patients seemed to be at borderline significantly increased risks of RCa (SIR:1.31; 95\% CI: 0.94-1.67; I²=48.0\%) and PCa (SIR: $1.13 ; 95 \%$ CI: $0.93-1.33 ; \mathrm{I}^{2}=73.5 \%$ ). Notably, we observed that IBD patients in Eastern countries have significantly increased PCa risk (SIR: 2.66; 95\% CI: 1.52-3.81; $\mathrm{I}^{2}=13.6 \%$ ), especially for UC patients (SIR: 3.01; 95\% CI: $\left.1.75-4.27 ; \mathrm{I}^{2}=0.0 \%\right)$.

Conclusions: Our findings indicate that IBD patients with special reference to CD patients increase the risk of RCa. Besides, IBD patients in Asian countries have significantly increased risk of PCa, especially for UC patients. Further studies are warranted to elucidate the potential mechanism of RCa associated with IBD and the differences of the risk of urinary cancers between Eastern and Western countries.

Keywords: Inflammatory bowel disease (IBD); bladder cancer; prostate cancer; renal cancer

Submitted Oct 21, 2020. Accepted for publication Jan 18, 2021.

doi: $10.21037 /$ tau-20-1358

View this article at: http://dx.doi.org/10.21037/tau-20-1358

^ ORCID: Dechao Feng, 0000-0002-8267-9920; Yubo Yang, 0000-0002-0189-3256; Wuran Wei, 0000-0002-2133-6043. 


\section{Introduction}

Inflammatory bowel disease (IBD), traditionally classified into Crohn's disease (CD) and ulcerative colitis (UC), is an idiopathic intestinal inflammatory disease involving the ileum, rectum, and colon (1). IBD is a multifactorial disorder, and it is well-known that the chronic inflammatory response caused by the abnormal reaction of the intestinal mucosal immune system plays an important role in the pathogenesis of IBD (1). In the latter half of the 20th century, IBD was traditionally considered as a disease of westernized nations (including the USA, Canada, Australia, New Zealand, and all countries in western Europe), due to the significantly increased incidence of UC and CD in the western world (2-4) However, recent studies indicate that IBD has become a global condition with the development of newly industrialized countries whose societies have become more westernized (including South America, eastern Europe, Asia, and Africa) (2,5). IBD has been demonstrated with an increased risk of intestinal and extra-intestinal tumors $(6-11)$. Although recent studies $(10,11)$ showed that IBD patients were at increased risk of bladder cancer $(\mathrm{BCa})$ and prostate cancer $(\mathrm{PCa})$, limited and disparate data were available for incidence of urological malignancies in these patients. To better understand this issue, a meta-analysis of population-based studies was performed to elucidate the risk of urologic cancers in patients with CD or UC. We present the following article in accordance with the PRISMA reporting checklist (available at http://dx.doi. org/10.21037/tau-20-1358).

\section{Methods}

\section{Search strategy}

In accordance with the Preferred Reporting Items for Systematic Reviews and Meta-Analyses (PRISMA) Statement (12), this literature was identified initially through PubMed, the Cochrane Library, Embase and Web of Science from inception to June 2020 with no language limitation. The Medical Subject Heading (MeSH) terms including "Inflammatory Bowel Diseases", "Urinary Bladder Neoplasms", "Prostatic Neoplasms", "Kidney Neoplasms", "Genital Neoplasms, Male" and "Urologic Neoplasms", and corresponding synonyms were combined in search strategy. The complete search strategy was presented in Supplementary Material. We scrutinized references of identified studies manually for all potentially eligible studies to broaden the search.

\section{Study selection}

We developed the following inclusion criteria to identify qualified documents: (I) After diagnosis of IBD, regardless of $\mathrm{UC}$ or $\mathrm{CD}$, patients were developed with $\mathrm{BCa}$, renal caner (RCa), $\mathrm{PCa}$, or male genital cancer; (II) the sample size included in this study is not less than 1,000; (III) standardized incidence ratio (SIR) or relative risk (RR), with corresponding $95 \%$ confidence intervals (CIs), were used to evaluate the association between IBD and risk of urologic cancers (BCa, RCa, PCa or MGCa); (IV) populationbased cohort studies or case-control studies; (V) the followup should more than one year after diagnosis of IBD. We incorporated the most recent or most informative study if more than one articles studied the same population. Besides, exclusion criteria were as follows: (I) any study which did not satisfy the inclusion criteria; (II) meeting abstracts, review or meta-analysis; (III) data not available. Figure 1 sketches the PRISMA flowchart showing the study selection process of this meta-analysis.

\section{Data extraction and quality assessment}

We imported the identified records into the EndNote X9. The initial search results were then screened by two independent authors using prespecified standardized criteria on the basis of title, abstract, and, finally, full text. Any discrepancy was tackled by consensus or a third party. We formulated a unified plan in advance to extract data. Data were independently extracted by two reviewers. The following data were extracted: the first author's name, year of publication, country, study design, period, sample size, cancer types, outcomes (SIRs or RRs).

The methodological quality of included studies was assessed by two independent reviewers using the NewcastleOttawa Scale (NOS) (13). The NOS applied a 'star system' to evaluate the quality of study from three perspectives: the selection of the studies, the comparability of studies, and the assessment of outcome. If seven or more of nine stars were received, the study was regarded as to be high-quality. Moreover, two reviewers independently rated the level of evidence of the included articles through the Oxford Centre for Evidence-Based Medicine criteria (14); this scale graded studies from strongest (level 1) to weakest (level 5) strength of evidence according to study design and data quality. 


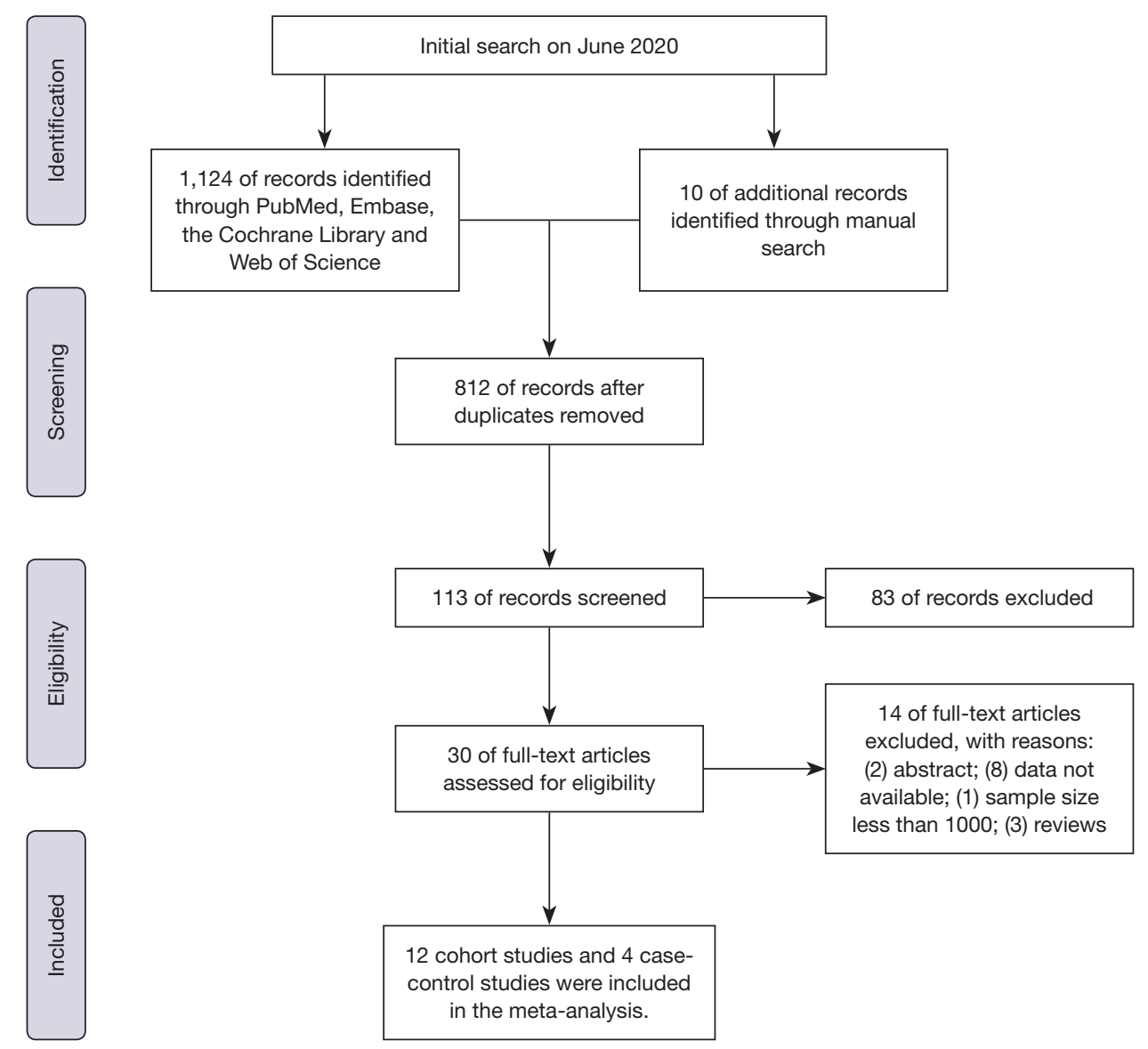

Figure 1 The PRISMA flowchart showing the study selection process of this meta-analysis.

\section{Statistical analysis}

The $\mathrm{Q}$ and $\mathrm{I}^{2}$-statistics tests were calculated to evaluate the between-study heterogeneity. Pooled SIRs or RRs and corresponding $95 \%$ CIs were calculated through fixed-effect model if the heterogeneity was acceptable among studies $\left(\mathrm{P}>0.1\right.$ or $\left.\mathrm{I}^{2} \leq 50 \%\right)$. Otherwise, a randomeffect model was used. Moreover, the results of this metaanalysis were reported on the basis of cancer types and IBD subtype. We assessed the potential publication bias according to the asymmetry of funnel plots, and the asymmetry can be quantified by the Egger's test and Begg's test. A P value of less than 0.1 was considered as significant publication bias. Furthermore, we performed sensitivity analyses to evaluate the robustness of the pooled results. Statistical significance was set at $\mathrm{P}<0.05$. This metaanalysis was completed by STATA version 14.2 (StataCorp LP, College Station, TX, USA).

\section{Results}

\section{Search results}

A total of 1,124 records were scrutinized through electronic database and manual search from inception to April 2020. 12 cohort studies (15-26) and 4 case-control studies (27-30) were eligible after screening on the basis of title, abstract, and full text (Figure 1). Ten studies were from Europe, four from North America and two from Asia in the present metaanalysis. A total of 241,969 participants were incorporated in the cohort studies. Specifically, ten studies with 174,094 patients reported on PCa risk, nine studies with 136,502 subjects reporting on BCa risk, nine studies with 94,133 patients reporting on RCa risk and six studies with 58,483 patients reporting on MGCa. Only four case-control articles were available for urologic cancers, including 4 for $\mathrm{PCa}, 2$ for $\mathrm{RCa}$ and 2 for BCa. There were 28,787 IBD patients and 306,380 IBD-free subjects in the case-control 
Table 1 The main characteristics of included studies in this meta-analysis

\begin{tabular}{|c|c|c|c|c|c|c|c|c|}
\hline Study & Country & Design & Period & Population & Cancer type & Outcomes & NOS & LoE \\
\hline $\begin{array}{l}\text { Jussila et al. } \\
2013\end{array}$ & Finland & Cohort & 2000-2010 & $\begin{array}{l}16,649 \cup C \text { and } \\
5,315 \mathrm{CD}\end{array}$ & $\begin{array}{l}\mathrm{RCa}, \mathrm{BCa} \text { and } \\
\mathrm{PCa}\end{array}$ & SIR & 7 & $2 b$ \\
\hline $\begin{array}{l}\text { Kappelman } \\
\text { et al. } 2014\end{array}$ & Denmark & Cohort & 1978-2010 & $\begin{array}{l}13,756 \mathrm{CD} \text { and } \\
35,152 \cup \mathrm{UC}\end{array}$ & $\mathrm{BCa}$ and $\mathrm{PCa}$ & SIR & 9 & $2 b$ \\
\hline Loo et al. 2019 & Canada & Cohort & 1998-2015 & $\begin{array}{l}20,644 \mathrm{CD}, 14,000 \text { UC } \\
\text { and } 1,341 \text { unspecified }\end{array}$ & $\mathrm{PCa}$ & SIR & 9 & $2 b$ \\
\hline $\begin{array}{l}\text { Ekbom et al. } \\
1991\end{array}$ & Sweden & Cohort & $1965-1983$ & $\begin{array}{l}1,655 \mathrm{CD} \text { and } \\
3,121 \cup \mathrm{UC}\end{array}$ & $\begin{array}{l}\mathrm{RCa}, \mathrm{BCa}, \mathrm{PCa} \\
\text { and } \mathrm{MGCa}\end{array}$ & SIR & 7 & $2 b$ \\
\hline $\begin{array}{l}\text { Jess et al. } \\
2013\end{array}$ & Denmark & Cohort & 1978-2010 & $\begin{array}{l}1,515 \cup C \text { and } \\
810 \mathrm{CD}\end{array}$ & $\mathrm{MGCa}$ and $\mathrm{PCa}$ & SIR & 8 & $2 b$ \\
\hline So et al. 2017 & China & Cohort & 1990-2016 & $\begin{array}{l}1,603 \cup C \text { and } \\
1,018 C D\end{array}$ & $\mathrm{RCa}$ and $\mathrm{PCa}$ & SIR & 7 & $2 b$ \\
\hline $\begin{array}{l}\text { Karlen et al. } \\
1999\end{array}$ & Sweden & Cohort & 1955-1989 & $1,547 \cup C$ & $\begin{array}{l}\mathrm{RCa}, \mathrm{BCa}, \mathrm{PCa} \\
\text { and } \mathrm{MGCa}\end{array}$ & SIR & 8 & $2 b$ \\
\hline $\begin{array}{l}\text { Bourrier et al. } \\
2015\end{array}$ & France & Cohort & 2004-2005 & $\begin{array}{l}11,759 \mathrm{CD} \text { and } \\
7,727 \cup \mathrm{C}\end{array}$ & $\mathrm{RCa}$ and $\mathrm{BCa}$ & SIR & 9 & $2 b$ \\
\hline $\begin{array}{l}\text { Burns et al. } \\
2018\end{array}$ & USA & $\begin{array}{l}\text { Case- } \\
\text { control }\end{array}$ & 1996-2017 & $\begin{array}{l}1,033 \text { IBD and } \\
9,306 \text { IBD-free }\end{array}$ & $\mathrm{PCa}$ & $\mathrm{HR}$ & 9 & $3 b$ \\
\hline $\begin{array}{l}\text { Bernstein et al. } \\
2001\end{array}$ & Canada & $\begin{array}{l}\text { Case- } \\
\text { control }\end{array}$ & 1984-1997 & $\begin{array}{l}\text { 6,027 IBD and } \\
5,529 \text { IBD-free }\end{array}$ & $\mathrm{RCa}$ and $\mathrm{BCa}$ & IRR & 9 & $3 b$ \\
\hline
\end{tabular}

CD, Crohn's disease; UC, ulcerative colitis; IBD, inflammatory bowel disease; RCa, renal cancer; BCa, bladder cancer; PCa, prostate cancer; MGCa, male genital cancer; SIR, standardized incidence ratio; RR, relative risk; HR, hazard ratio; IRR, incidence rate ratio; NOS, Newcastle-Ottawa le; LoE, level of evidence.

studies. Table 1 presents the main characteristics of included studies in this meta-analysis.

\section{Meta-analysis results}

Standardized incidence ratio (SIR)

Nine of 12 cohort studies reported on $\mathrm{RCa}$ in patients with IBD, showing a significantly increased risk of RCa (pooled SIR: 1.53; 95\% CI: $1.25-1.80 ; \mathrm{I}^{2}=42.4 \%$; Figure 2). In the subgroup analysis, $\mathrm{RCa}$ risk was higher in patients with CD (pooled SIR: 1.95; 95\% CI: $1.45-2.44 ; I^{2}=39.9 \%$; Figure 2), and a borderline significantly increased risk of RCa was observed in patients with UC (pooled SIR: 1.31; 95\% CI:0.94-1.67; $\mathrm{I}^{2}=48.0 \%$; Figure 2) when compared to 


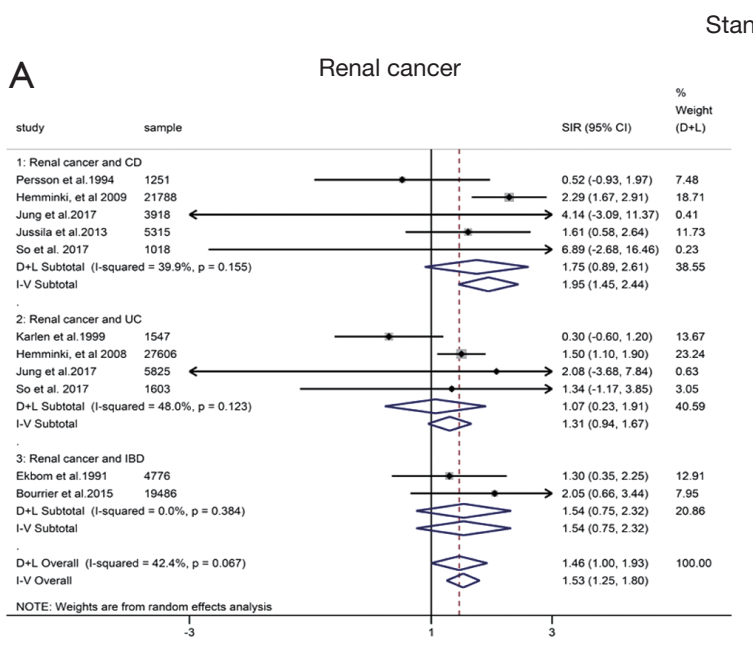

tandardized incidence ratio
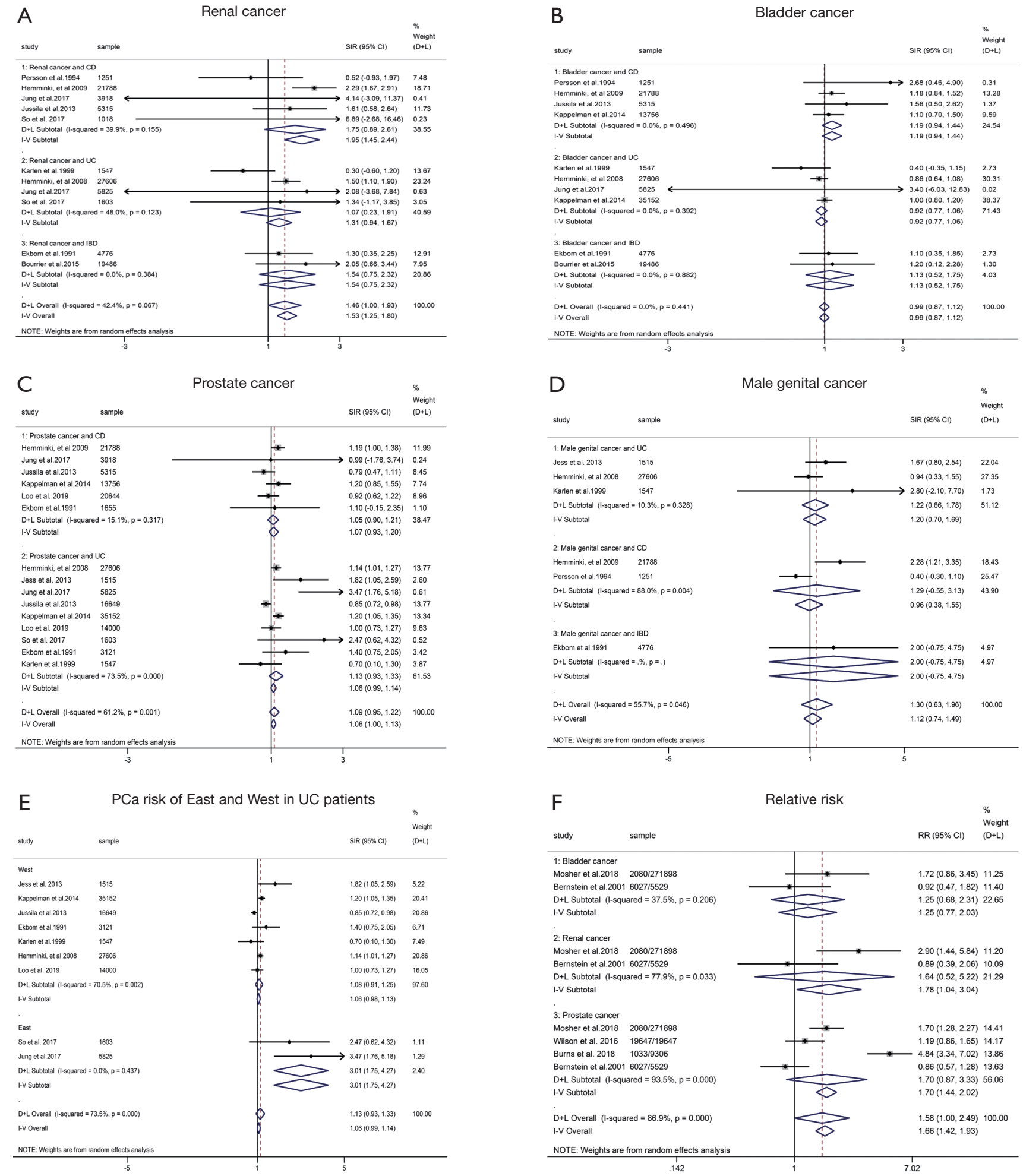

Figure 2 The pooled outcomes included in this meta-analysis. (A) renal cancer; (B) bladder cancer; (C) prostate cancer; (D) male genital cancer; (E) PCa risk of East and West in UC patients; (F) the pooled outcomes of included studies in terms of relative risk. PCa, prostate cancer; UC, ulcerative colitis. 
the background population. We observed that the risk of $\mathrm{PCa}$ in IBD patients were of borderline significant increase (pooled SIR:1.09; 95\% CI: 0.95-1.22; I ${ }^{2}=61.2 \%$; Figure 2) when compared with the background population, as was the risk of UC subgroup (pooled SIR: 1.13; 95\% CI: 0.93-1.33; $\mathrm{I}^{2}=73.5 \%$; Figure 2) and CD subgroup (pooled SIR: 1.07; 95\% CI: 0.93-1.20; $\mathrm{I}^{2}=15.1 \%$; Figure 2). Besides, we found that no significantly decreased risk of $\mathrm{BCa}$ was detected in patients with IBD (pooled SIR:0.99; 95\% CI: 0.87-1.12; $\mathrm{I}^{2}=0 \%$; Figure 2) and UC subgroup (pooled SIR: 0.92; $95 \%$ CI: $0.77-1.06$; $\mathrm{I}^{2}=0 \%$; Figure 2), whereas a trend toward an increased risk of $\mathrm{BCa}$ was observed in patients with $\mathrm{CD}$ subgroup (pooled SIR:1.19; 95\% CI: 0.94-1.44; $\mathrm{I}^{2}=0 \%$; Figure 2). Data from six cohort studies (18-20,24,27,30) showed that IBD or IBD subtype (UC and CD) did not significantly increase the risk of MGCa. The pooled SIRs were $1.30,1.20,1.29$ for IBD, UC and CD, respectively.

\section{Relative risk (RR)}

Only two case-control studies reported the results of RCa risk, resulting no significant difference between IBD group and IBD-free group (pooled RR: 1.64; 95\% CI: 0.52-5.22; $\mathrm{I}^{2}=77.9 \%$; Figure 2). Similarly, the two studies provided data on BCa in UC and CD combined, revealing that IBD patients seemed to have a higher risk of BCa than IBD-free subjects (RR: 1.25 ; 95\% CI: 0.77-2.03; $\mathrm{I}^{2}=37.5 \%$; Figure 2). Pooled analysis of four case-control studies (27-30) showed there was no significant difference between IBD group and IBD-free group regarding the PCa risk (RR: 1.70; 95\% CI:0.87-3.33; Figure 2), while there was significant heterogeneity between studies $\left(\mathrm{I}^{2}=93.5 \% ; \mathrm{P}=0.000\right)$. Subgroup analysis was unlikely to be conducted due to insufficient information.

\section{Publication bias and sensitivity analysis}

The Egger's test and Begg's test were used to quantify potential publication bias. In cohort studies, the $\mathrm{p}$ values of Egger's test and Begg's test were 0.865 and 0.213 for IBD patients with $\mathrm{RCa}$, respectively. Besides, $\mathrm{p}$ values of Egger's test for PCa, BCa and MGCa were 0.234, 0.194 and 0.276 , respectively. In case-control studies, the $p$ values of Egger's test and Begg's test were 0.078 and 0.108 for patients with urologic cancers, respectively. Thus, we concluded that there was no significant publication bias in this study. Furthermore, we performed a sensitivity analysis to assess the impact of a single study on the overall effect size through removing each study from the meta-analysis sequentially (Figure 3). As a result, no noticeable changes were observed.

\section{Discussion}

In the last century, IBD was mainly concentrated in western developed countries, and little was known about the number of individuals influenced by IBD outside the western world (2). At the turn of the 21st century, newer epidemiological studies found a rapid increase in IBD incidence among newly industrialized countries in Asia, including China and India $(2,5)$. This epidemiological shift occurring with newly industrialized and urbanized countries reflecting the experience reported in the west more than 50 years ago (2). IBD including CD and $\mathrm{UC}$ is associated with increased risk of intestinal and extraintestinal cancers (6-11). Local and systemic inflammation were postulated to contribute to the increased risk of cancer in IBD patients (31). Given scarce population-based data about the risk of urinary tumors, we conducted this meta-analysis to illuminate the relationship between IBD and risk of urologic cancers to provide patient consultation and guide clinical practice.

The previous two meta-analysis $(10,11)$ had controversial results regarding PCa risk and UC patients. Pedersen et al. (10) pooled analysis of 4 studies and found that UC patients were not at increased risk of PCa (SIR: 1.14; 95\% CI: 0.85-1.52). Conversely, Ge et al. (11) observed IBD patients have significantly elevated PCa risk (SIR: 1.33; 95\% CI: $1.03-1.71$ ), especially for UC patients (SIR: 1.58; $95 \%$ CI: 1.08-2.30). In the present meta-analysis, pooled data from ten cohort studies indicated that IBD patients were at borderline significantly increased risk of $\mathrm{PCa}$, regardless of UC or CD patients. The difference above might be attributed to geographic region. The 4 studies included in Pedersen's article were from Western countries, while 2 of 5 studies included in Ge's article were from Asian countries. Thus, we conducted a subgroup according to geographic region. We observed that IBD patients in Eastern countries have significantly increased risk of PCa (SIR: 2.66; 95\% CI: $1.52-3.81 ; \mathrm{I}^{2}=13.6 \%$ ), especially for UC patients (SIR: 3.01; 95\% CI: $1.75-4.27 ; \mathrm{I}^{2}=0.0 \%$; Figure 2). Overall, the incidence of IBD in Asian countries remains lower than that in Western countries, while the rapid increase in disease incidence will also aggravate socioeconomic burden in Asian countries (21). The difference of environmental backgrounds and genetic susceptibility in IBD patients between Asian and Western countries might explain the 
Standardized incidence ratio

A

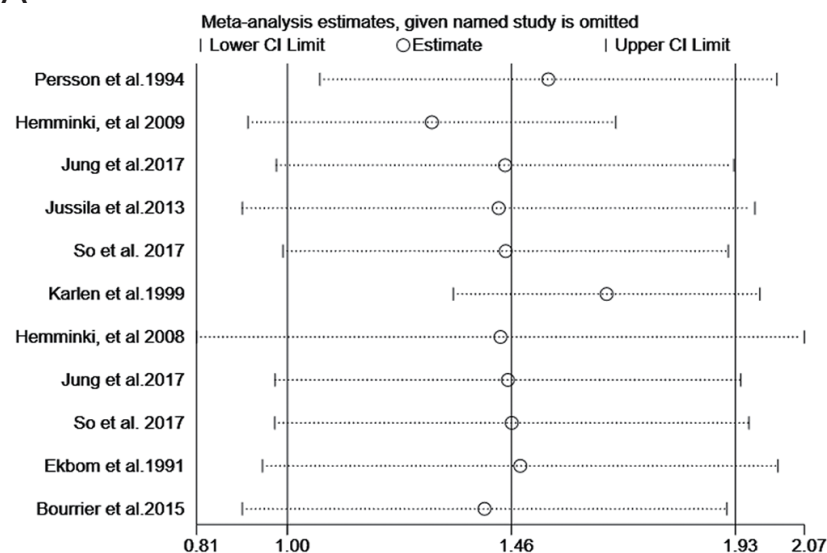

C

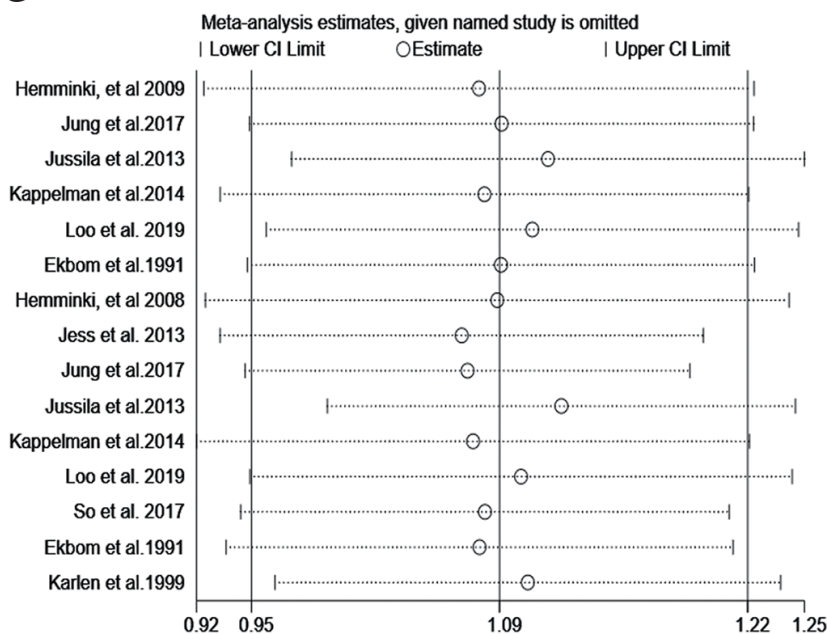

E

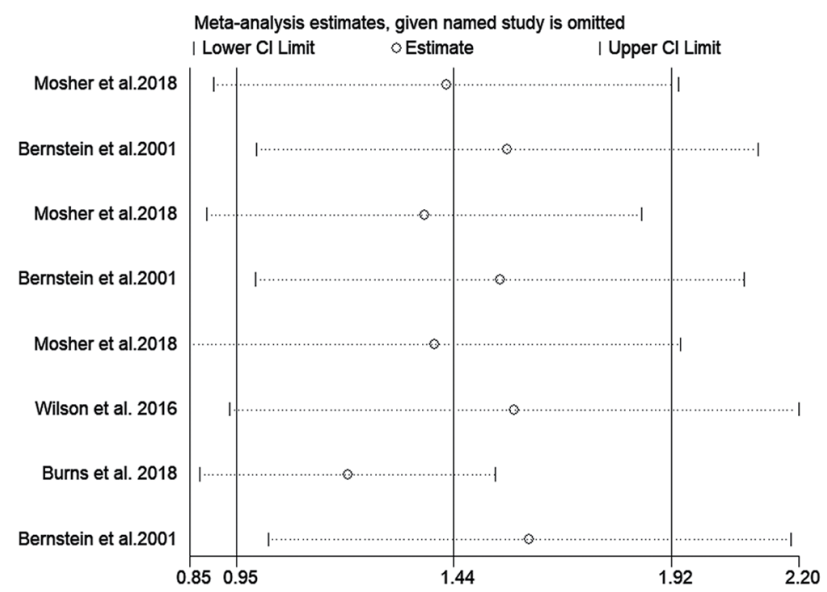

B

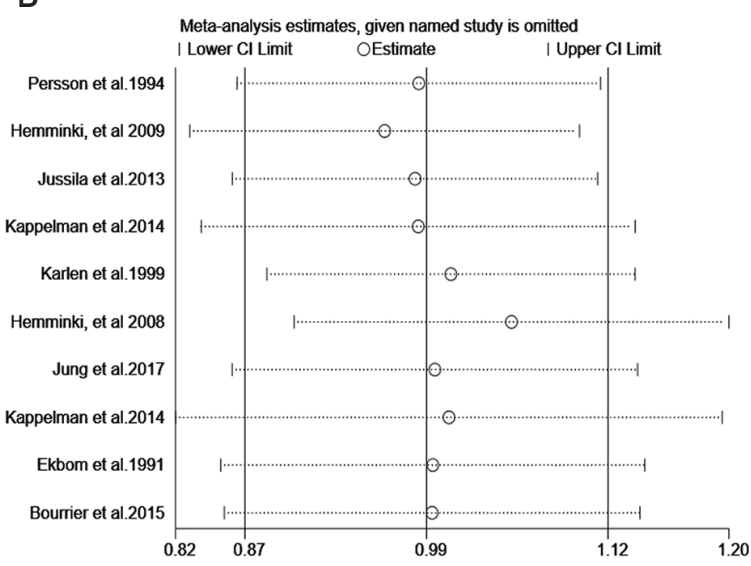

D

Male genital cancer

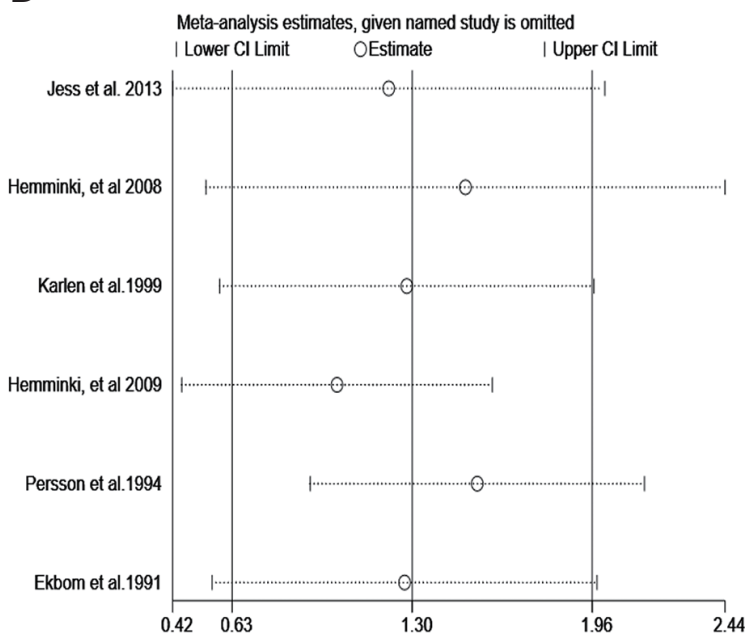

Relative risk

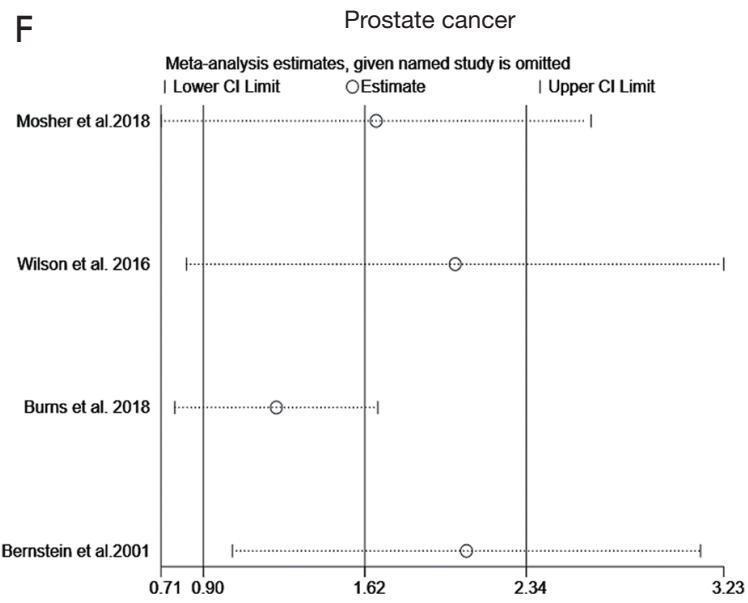

Figure 3 The sensitivity analyses of outcomes in this meta-analysis. (A) renal cancer; (B) bladder cancer; (C) prostate cancer; (D) male genital cancer; (E) the sensitivity analysis of urinary cancers with respect to relative risk; (F) the sensitivity analysis of prostate cancer with respect to prostate cancer. 
predisposition of PCa in Eastern patients with IBD.

Unlike the previous meta-analysis (10), our meta-analysis did not observe a significant increase of $\mathrm{BCa}$ risk in $\mathrm{CD}$ patients. We thought that the sample size and statistical methods contributed to this difference. Pedersen et al. (10) pooled data with less sample size and some data were calculated as the number of person-years of observation divided by the number of patients in the cohort, which might be inappropriate. Besides, our meta-analysis detected that IBD patients, especially CD patients, increased the risk of $\mathrm{RCa}$. When we restricted the analysis to Western countries (data not shown), the pooled data were in accordance with the previous meta-analysis (100. However, due to the limited studies and defective statistical methods in the previous study (10), we were unlikely to make a further conclusion. Similar to the previous study (10), our meta-analysis did not observe an increased MGCa risk in IBD patients. However, all studies pooled for MGCa were from Western countries. Data reporting MGCa risk and IBD patients are still insufficient for Eastern countries.

The use of immunosuppressive or biologic agents might have opposite effects in risk of intestinal and extraintestinal cancers. The widespread use of immunosuppressive or biologic agents may reduce the risk of colorectal cancer by suppressing intestinal inflammation (32), but contribute to the development of extraintestinal cancers (9,33-36). In IBD patients exposed to azathioprine, Pasternak et al. (37) firstly reported that IBD patients with azathioprine were more prone to have urinary tract cancer (UTC) than no users. In the present study, only one study (16) reported the association between thiopurine therapy and risk of urinary cancers in IBD patients. They found that IBD patients receiving thiopurines have a higher risk of UTC than those not receiving thiopurines (Hazard ratio: 2.82; 95\% CI: 1.047.68; $\mathrm{P}=0.04$ ) (16). However, they were unable to assess the impact of IBD patients with immunosuppressive therapy prone to undergo imaging techniques of the abdomen, smoking status, and anti-tumor necrosis factor on UTC risk. Therefore, the current evidence on immunosuppressive agents and the risk of UTC is still vastly limited. Despite this, they still advocated an imaging screening strategy (ultrasound or computerized tomography) before initiation of immunosuppressive therapy in elderly patients, especially for male smokers, due to the high prevalence of UTC in this population and the potential facilitating effect of thiopurines on tumor growth $(16,38)$. Besides, we are also unable to estimate other independent risk factors for UTC in patients with IBD as a result of insufficient data. Furthermore, although the rapidly increase in IBD incidence is accelerating the globalization of this disease, epidemiological studies reporting the relationship between IBD and UTI risk are still penurious.

Taken together, our study has preliminarily explored the relationship between IBD patients and risk of urinary cancers, providing a reference for cancer counseling and screening strategy for clinical management of these patients. With the westernization and industrialization of emerging countries, clinicians, especially in outpatient, need to raise awareness of screening patients with IBD for urinary tumors.

\section{Conclusions}

Our findings indicate that IBD patients with special reference to $\mathrm{CD}$ patients increase the risk of $\mathrm{RCa}$. Besides, IBD patients in Asian countries have significantly increased risk of PCa, especially for UC patients. Further studies are warranted to elucidate the potential mechanism of $\mathrm{RCa}$ associated with IBD and the differences of the risk of urinary cancers between Eastern and Western countries.

\section{Acknowledgments}

We thank Prof. Gong Juan from the College of Foreign Languages, Sichuan University for her contribution to editing the language of this study.

Funding: This work was supported by Department of Science and Technology of Sichuan Province (2020YFH0099) and the National Natural Science Foundation of China (No. 81370272, 30901621/C1705). The funders had no role in study design, data collection and analysis, decision to publish, or preparation of the manuscript.

\section{Footnote}

Reporting Checklist: The authors have completed the PRISMA reporting checklist. Available at http://dx.doi. org/10.21037/tau-20-1358

Conflicts of Interest: All authors have completed the ICMJE uniform disclosure form (available at http://dx.doi. org/10.21037/tau-20-1358). The authors have no conflicts of interest to declare.

Ethical Statement: The authors are accountable for all 
aspects of the work in ensuring that questions related to the accuracy or integrity of any part of the work are appropriately investigated and resolved.

Open Access Statement: This is an Open Access article distributed in accordance with the Creative Commons Attribution-NonCommercial-NoDerivs 4.0 International License (CC BY-NC-ND 4.0), which permits the noncommercial replication and distribution of the article with the strict proviso that no changes or edits are made and the original work is properly cited (including links to both the formal publication through the relevant DOI and the license). See: https://creativecommons.org/licenses/by-nc-nd/4.0/.

\section{References}

1. Baumgart DC, Carding SR. Inflammatory bowel disease: cause and immunobiology. Lancet 2007;369:1627-40.

2. Ng SC, Shi HY, Hamidi N, et al. Worldwide incidence and prevalence of inflammatory bowel disease in the 21 st century: a systematic review of population-based studies. Lancet 2017;390:2769-78.

3. Kaplan GG, Ng SC. Understanding and preventing the global increase of inflammatory bowel disease. Gastroenterology 2017;152:313-21.e2.

4. Molodecky NA, Soon IS, Rabi DM, et al. Increasing incidence and prevalence of the inflammatory bowel diseases with time, based on systematic review. Gastroenterology 2012;142:46-54.e42.

5. Mak WY, Zhao M, Ng SC, et al. The epidemiology of inflammatory bowel disease: East meets west. J Gastroenterol Hepatol 2020;35:380-9.

6. Lutgens MW, van Oijen MG, van der Heijden GJ, et al. Declining risk of colorectal cancer in inflammatory bowel disease: an updated meta-analysis of population-based cohort studies. Inflamm Bowel Dis 2013;19:789-99.

7. Huang SZ, Liu ZC, Liao WX, et al. Risk of skin cancers in thiopurines-treated and thiopurines-untreated patients with inflammatory bowel disease: A systematic review and meta-analysis. J Gastroenterol Hepatol 2019;34:507-16.

8. Singh S, Nagpal SJ, Murad MH, et al. Inflammatory bowel disease is associated with an increased risk of melanoma: a systematic review and meta-analysis. Clin Gastroenterol Hepatol 2014;12:210-8.

9. Kandiel A, Fraser AG, Korelitz BI, et al. Increased risk of lymphoma among inflammatory bowel disease patients treated with azathioprine and 6- mercaptopurine. Gut 2005;54:1121-5.
10. Pedersen N, Duricova D, Elkjaer M, et al. Risk of extraintestinal cancer in inflammatory bowel disease: metaanalysis of population-based cohort studies. Am J Gastroenterol 2010;105:1480-7.

11. Ge Y, Shi Q, Yao W, et al. The association between inflammatory bowel disease and prostate cancer risk: a meta-analysis. Prostate Cancer Prostatic Dis 2020;23:53-8.

12. Moher D, Liberati A, Tetzlaff J, et al. Preferred reporting items for systematic reviews and meta-analyses: the PRISMA statement. PLoS Med 2009;6:e1000097.

13. Wells GA, Shea B, O'Connell D, et al. The NewcastleOttawa Scale (NOS) for assessing the quality if nonrandomized studies in meta-analyses. Ottawa Hospital Research Institute. Available online: www.ohri.ca/ programs/clinical_epidemiology/oxford.asp

14. Evidence-Based Cf. Oxford Centre for evidence-based medicine: levels of evidence, 2009. Available online: http:// www.cebm.net/oxford-centreevidence-based-medicinelevels-evidence-march-2009/

15. Bernstein CN, Blanchard JF, Kliewer E, et al. Cancer risk in patients with inflammatory bowel disease: a populationbased study. Cancer 2001;91:854-62.

16. Bourrier A, Carrat F, Colombel JF, et al. Excess risk of urinary tract cancers in patients receiving thiopurines for inflammatory bowel disease: a prospective observational cohort study. Aliment Pharmacol Ther 2016;43:252-61.

17. Burns JA, Weiner AB, Catalona WJ, et al. Inflammatory bowel disease and the risk of prostate cancer. Eur Urol 2019;75:846-52.

18. Hemminki K, Li X, Sundquist J, et al. Cancer risks in ulcerative colitis patients. Int J Cancer 2008;123:1417-21.

19. Hemminki K, Li X, Sundquist J, et al. Cancer risks in Crohn disease patients. Ann Oncol 2009;20:574-80.

20. Jess T, Horvath-Puho E, Fallingborg J, et al. Cancer risk in inflammatory bowel disease according to patient phenotype and treatment: a Danish population-based cohort study. Am J Gastroenterol 2013;108:1869-76.

21. Jung YS, Han M, Park S, et al. Cancer risk in the early stages of inflammatory bowel disease in Korean patients: a nationwide population-based study. J Crohns Colitis 2017;11:954-62.

22. Jussila A, Virta LJ, Pukkala E, et al. Malignancies in patients with inflammatory bowel disease: a nationwide register study in Finland. Scand J Gastroenterol 2013;48:1405-13.

23. Kappelman MD, Farkas DK, Long MD, et al. Risk of cancer in patients with inflammatory bowel diseases: a nationwide population-based cohort study with 30 years 
of follow-up evaluation. Clin Gastroenterol Hepatol 2014;12:265-73.e1.

24. Karlén P, Loferg R, Brostrom O, et al. Increased risk of cancer in ulcerative colitis: a population-based cohort study. Am J Gastroenterol 1999;94:1047-52.

25. Loo SY, Maria V, Alain B, et al. Risk of Malignant Cancers in Inflammatory Bowel Disease. J Crohns Colitis 2019;13:1302-10.

26. Mosher CA, Brown GR, Weideman RA, et al. Incidence of colorectal cancer and extracolonic cancers in veteran patients with inflammatory bowel disease. Inflamm Bowel Dis 2018;24:617-23.

27. Persson PG, Karlen P, Bernell O, et al. Crohn's disease and cancer: a population-based cohort study. Gastroenterology 1994;107:1675-9.

28. So J, Tang W, Leung WK, et al. Cancer risk in 2621 Chinese patients with inflammatory bowel disease: a population-based cohort study. Inflamm Bowel Dis 2017;23:2061-8.

29. Wilson JC, Furlano RI, Jick SS, et al. A populationbased study examining the risk of malignancy in patients diagnosed with inflammatory bowel disease. J Gastroenterol 2016;51:1050-62.

30. Ekbom A, Helmick C, Zack M, et al. Extracolonic malignancies in inflammatory bowel disease. Cancer 1991;67:2015-9.

31. Mak JWY, So J, Tang W, et al. Cancer risk and chemoprevention in Chinese inflammatory bowel disease patients: a population-based cohort study. Scand J Gastroenterol 2020;55:279-86.

32. Andersen NN, Jess T. Has the risk of colorectal cancer in inflammatory bowel disease decreased? World J Gastroenterol 2013;19:7561-8.

33. Beaugerie L, Brousse N, Bouvier AM, et al. Lymphoproliferative disorders in patients receiving thiopurines for inflammatory bowel disease: a prospective observational cohort study. Lancet 2009;374:1617-25.

34. Armstrong RG, West J, Card TR. Risk of cancer in inflammatory bowel disease treated with azathioprine: a UK population-based case-control study. Am J Gastroenterol 2010;105:1604-9.

35. Peyrin-Biroulet L, Khosrotehrani K, Carrat F, et al. Increased risk for nonmelanoma skin cancers in patients who receive thiopurines for inflammatory bowel disease. Gastroenterology 2011;141:1621-1628.e1621-1625.

36. Long MD, Martin CF, Pipkin CA, et al. Risk of melanoma and nonmelanoma skin cancer among patients with inflammatory bowel disease. Gastroenterology 2012;143:390-399.e1.

37. Pasternak B, Svanström H, Schmiegelow K, et al. Use of azathioprine and the risk of cancer in inflammatory bowel disease. Am J Epidemiol 2013;177:1296-305.

38. Gutierrez-Dalmau A, Campistol JM. Immunosuppressive therapy and malignancy in organ transplant recipients: a systematic review. Drugs 2007;67:1167-98.
Cite this article as: Feng D, Yang Y, Wang Z, Wei W, Li L. Inflammatory bowel disease and risk of urinary cancers: a systematic review and pooled analysis of population-based studies. Transl Androl Urol 2021;10(3):1332-1341. doi: 10.21037/ tau-20-1358 


\section{Inflammatory Bowel Diseases and bladder cancer}

PubMed

((((Inflammatory Bowel Diseases[Title/Abstract]) OR Inflammatory Bowel Disease[Title/Abstract]) OR Bowel Diseases, Inflammatory[Title/Abstract])) AND $(((()(((()(((()(($ Urinary Bladder Neoplasms[Title/Abstract]) OR Neoplasm, Urinary Bladder[Title/Abstract]) OR Urinary Bladder Neoplasm[Title/Abstract]) OR Neoplasms, Bladder[Title/Abstract]) OR Bladder Neoplasms[Title/Abstract]) OR Bladder Neoplasm[Title/Abstract]) OR Neoplasm, Bladder[Title/Abstract]) OR Bladder Tumors[Title/Abstract]) OR Bladder Tumor[Title/Abstract]) OR Tumor, Bladder[Title/Abstract]) OR Tumors, Bladder[Title/Abstract]) OR Urinary Bladder Cancer[Title/Abstract]) OR Cancer, Urinary Bladder[Title/Abstract]) OR Malignant Tumor of Urinary Bladder[Title/Abstract]) OR Cancer of the Bladder[Title/Abstract]) OR Bladder Cancer[Title/ Abstract]) OR Bladder Cancers[Title/Abstract]) OR Cancer, Bladder[Title/Abstract]) OR Cancer of Bladder[Title/Abstract]) 44 records

\section{Web of Science}

\#1 TOPIC: (Inflammatory Bowel Diseases) OR TOPIC: (Inflammatory Bowel Disease) OR TOPIC: (Bowel Diseases, Inflammatory)

\#2 TOPIC: (Urinary Bladder Neoplasms) OR TOPIC: (Neoplasm, Urinary Bladder) OR TOPIC: (Urinary Bladder Neoplasm) OR TOPIC: (Neoplasms, Bladder) OR TOPIC: (Bladder Neoplasms) OR TOPIC: (Bladder Neoplasm) OR TOPIC: (Neoplasm, Bladder) OR TOPIC: (Bladder Tumors) OR TOPIC: (Bladder Tumor) OR TOPIC: (Tumor, Bladder) OR TOPIC: (Tumors, Bladder) OR TOPIC: (Urinary Bladder Cancer) OR TOPIC: (Cancer, Urinary Bladder) OR TOPIC: (Malignant Tumor of Urinary Bladder) OR TOPIC: (Cancer of the Bladder) OR TOPIC: (Bladder Cancer) OR TOPIC: (Bladder Cancers) OR TOPIC: (Cancer, Bladder) OR TOPIC: (Cancer of Bladder)

\#3 \#1 and \#2 115 records

\section{OVID}

\#1 (Inflammatory Bowel Diseases or Inflammatory Bowel Disease or Bowel Diseases, Inflammatory).kw.

\#2 (Urinary Bladder Neoplasms or Neoplasm, Urinary Bladder or Urinary Bladder Neoplasm or Neoplasms, Bladder or Bladder Neoplasms or Bladder Neoplasm or Neoplasm, Bladder or Bladder Tumors or Bladder Tumor or Tumor, Bladder or Tumors, Bladder or Urinary Bladder Cancer or Cancer, Urinary Bladder or Malignant Tumor of Urinary Bladder or Cancer of the Bladder or Bladder Cancer or Bladder Cancers or Cancer, Bladder or Cancer of Bladder).kw.

\#3 \#1 and \#2 3 records

\section{Inflammatory Bowel Diseases and renal cancer}

((((Inflammatory Bowel Diseases[Title/Abstract]) OR Inflammatory Bowel Disease[Title/Abstract]) OR Bowel Diseases, Inflammatory[Title/Abstract])) AND ((()((()((()((((Kidney Neoplasms[Title/Abstract]) OR Kidney Neoplasm[Title/Abstract]) OR Neoplasm, Kidney[Title/Abstract]) OR Renal Neoplasms[Title/Abstract]) OR Neoplasm, Renal[Title/Abstract]) OR Neoplasms, Renal[Title/Abstract]) OR Renal Neoplasm[Title/Abstract]) OR Neoplasms, Kidney[Title/Abstract]) OR Cancer of Kidney[Title/Abstract]) OR Kidney Cancers[Title/Abstract]) OR Renal Cancer[Title/Abstract]) OR Cancer, Renal[Title/ Abstract]) OR Cancers, Renal[Title/Abstract]) OR Renal Cancers[Title/Abstract]) OR Cancer of the Kidney[Title/Abstract]) OR Kidney Cancer[Title/Abstract]) OR Cancer, Kidney[Title/Abstract]) OR Cancers, Kidney[Title/Abstract]) 101 records

\section{OVID}

\#1 (Inflammatory Bowel Diseases or Inflammatory Bowel Disease or Bowel Diseases, Inflammatory).kw.

\#2 (Kidney Neoplasms or Kidney Neoplasm or Neoplasm, Kidney or Renal Neoplasms or Neoplasm, Renal or Neoplasms, Renal or Renal Neoplasm or Neoplasms, Kidney or Cancer of Kidney or Kidney Cancers or Renal Cancer or Cancer, Renal 
or Cancers, Renal or Renal Cancers or Cancer of the Kidney or Kidney Cancer or Cancer, Kidney or Cancers, Kidney).kw. \#3 \#1 and \#2 1 record

\section{Web of Science}

\#1 TOPIC: (Inflammatory Bowel Diseases) OR TOPIC: (Inflammatory Bowel Disease) OR TOPIC: (Bowel Diseases, Inflammatory)

\#2 TOPIC: (Kidney Neoplasms) OR TOPIC: (Kidney Neoplasm) OR TOPIC: (Neoplasm, Kidney) OR TOPIC: (Renal Neoplasms) OR TOPIC: (Neoplasm, Renal) OR TOPIC: (Neoplasms, Renal) OR TOPIC: (Renal Neoplasm) OR TOPIC: (Neoplasms, Kidney) OR TOPIC: (Cancer of Kidney) OR TOPIC: (Kidney Cancers) OR TOPIC: (Renal Cancer) OR TOPIC: (Cancer, Renal) OR TOPIC: (Cancers, Renal) OR TOPIC: (Renal Cancers) OR TOPIC: (Cancer of the Kidney) OR TOPIC: (Kidney Cancer) OR TOPIC: (Cancer, Kidney) OR TOPIC: (Cancers, Kidney)

\#3 \#1 and \#2 312 records

\section{Inflammatory Bowel Diseases and prostate cancer}

\section{Pub}

(((()((()((()((((Prostatic Neoplasms[Title/Abstract]) OR Prostate Neoplasms[Title/Abstract]) OR Neoplasms, Prostate[Title/ Abstract]) OR Neoplasm, Prostate[Title/Abstract]) OR Prostate Neoplasm[Title/Abstract]) OR Neoplasms, Prostatic[Title/ Abstract]) OR Neoplasm, Prostatic[Title/Abstract]) OR Prostatic Neoplasm[Title/Abstract]) OR Prostate Cancer[Title/ Abstract]) OR Cancer, Prostate[Title/Abstract]) OR Cancers, Prostate[Title/Abstract]) OR Prostate Cancers[Title/Abstract]) OR Cancer of the Prostate[Title/Abstract]) OR Prostatic Cancer[Title/Abstract]) OR Cancer, Prostatic[Title/Abstract]) OR Cancers, Prostatic[Title/Abstract]) OR Prostatic Cancers[Title/Abstract]) OR Cancer of Prostate[Title/Abstract])) AND (((Inflammatory Bowel Diseases[Title/Abstract]) OR Inflammatory Bowel Disease[Title/Abstract]) OR Bowel Diseases, Inflammatory[Title/Abstract]) 109 records

\section{Web}

TOPIC: $(((((()((((()(((($ Prostatic Neoplasms) OR Prostate Neoplasms) OR Neoplasms, Prostate) OR Neoplasm, Prostate) OR Prostate Neoplasm) OR Neoplasms, Prostatic) OR Neoplasm, Prostatic) OR Prostatic Neoplasm) OR Prostate Cancer) OR Cancer, Prostate) OR Cancers, Prostate) OR Prostate Cancers) OR Cancer of the Prostate) OR Prostatic Cancer) OR Cancer, Prostatic) OR Cancers, Prostatic) OR Prostatic Cancers) OR Cancer of Prostate)) AND (((Inflammatory Bowel Diseases) OR Inflammatory Bowel Disease) OR Bowel Diseases, Inflammatory)) 305 records

\section{OVID}

\#1 (Prostatic Neoplasms or Prostate Neoplasms or Neoplasms, Prostate or Neoplasm, Prostate or Prostate Neoplasm or Neoplasms, Prostatic or Neoplasm, Prostatic or Prostatic Neoplasm or Prostate Cancer or Cancer, Prostate or Cancers, Prostate or Prostate Cancers or Cancer of the Prostate or Prostatic Cancer or Cancer, Prostatic or Cancers, Prostatic or Prostatic Cancers or Cancer of Prostate).kw.

\#2 (Inflammatory Bowel Diseases or Inflammatory Bowel Disease or Bowel Diseases, Inflammatory).kw.

\#3 1 and 222 records

\section{Inflammatory Bowel Disease and male genital cancer}

\section{Pub}

((((((Genital Neoplasms, Male[Title/Abstract]) OR Neoplasms, Male Genital[Title/Abstract]) OR Male Genital Neoplasms[Title/Abstract]) OR Genital Neoplasm, Male[Title/Abstract]) OR Male Genital Neoplasm[Title/Abstract]) OR Neoplasm, Male Genital[Title/Abstract])) AND (((Inflammatory Bowel Diseases[Title/Abstract]) OR Inflammatory Bowel 
Disease[Title/Abstract]) OR Bowel Diseases, Inflammatory[Title/Abstract]) 3 records

Web

(((((((Genital Neoplasms, Male) OR Neoplasms, Male Genital) OR Male Genital Neoplasms) OR Genital Neoplasm, Male) OR Male Genital Neoplasm) OR Neoplasm, Male Genital)) AND (((Inflammatory Bowel Diseases) OR Inflammatory Bowel Disease) OR Bowel Diseases, Inflammatory)) 1 records

OVID 0 records

\section{Inflammatory Bowel Disease and urological tumors}

OVID

\#1 (Inflammatory Bowel Diseases or Inflammatory Bowel Disease or Bowel Diseases, Inflammatory).kw

\#2 (Urologic Neoplasms or Urological Neoplasms or Neoplasm, Urological or Neoplasms, Urological or Urological Neoplasm or Urinary Tract Neoplasms or Neoplasm, Urinary Tract or Neoplasms, Urinary Tract or Tract Neoplasm, Urinary or Tract Neoplasms, Urinary or Urinary Tract Neoplasm or Neoplasms, Urologic or Neoplasm, Urologic or Urologic Neoplasm or Cancer of Urinary Tract or Urinary Tract Cancers or Urological Cancer or Cancer, Urological or Cancers, Urological or Urological Cancers or Cancer of the Urinary Tract or Urinary Tract Cancer or Cancer, Urinary Tract or Cancers, Urinary Tract or Urologic Cancer or Cancer, Urologic or Cancers, Urologic or Urologic Cancers).kw.

\#3 \#1 and \#2 0 records

\section{PubMed}

((((Inflammatory Bowel Diseases[Title/Abstract]) OR Inflammatory Bowel Disease[Title/Abstract]) OR Bowel Diseases, Inflammatory[Title/Abstract])) AND $((()((()((()((()((()(()((($ Urologic Neoplasms[Title/Abstract]) OR Urological Neoplasms[Title/Abstract]) OR Neoplasm, Urological[Title/Abstract]) OR Neoplasms, Urological[Title/Abstract]) OR Urological Neoplasm[Title/Abstract]) OR Urinary Tract Neoplasms[Title/Abstract]) OR Neoplasm, Urinary Tract[Title/ Abstract]) OR Neoplasms, Urinary Tract[Title/Abstract]) OR Tract Neoplasm, Urinary[Title/Abstract]) OR Tract Neoplasms, Urinary[Title/Abstract]) OR Urinary Tract Neoplasm[Title/Abstract]) OR Neoplasms, Urologic[Title/Abstract]) OR Neoplasm, Urologic[Title/Abstract]) OR Urologic Neoplasm[Title/Abstract]) OR Cancer of Urinary Tract[Title/ Abstract]) OR Urinary Tract Cancers[Title/Abstract]) OR Urological Cancer[Title/Abstract]) OR Cancer, Urological[Title/ Abstract]) OR Cancers, Urological[Title/Abstract]) OR Urological Cancers[Title/Abstract]) OR Cancer of the Urinary Tract[Title/Abstract]) OR Urinary Tract Cancer[Title/Abstract]) OR Cancer, Urinary Tract[Title/Abstract]) OR Cancers, Urinary Tract[Title/Abstract]) OR Urologic Cancer[Title/Abstract]) OR Cancer, Urologic[Title/Abstract]) OR Cancers, Urologic[Title/Abstract]) OR Urologic Cancers[Title/Abstract]) 40 records

\section{Web of Science}

\#1 TOPIC: (Inflammatory Bowel Diseases) OR TOPIC: (Inflammatory Bowel Disease) OR TOPIC: (Bowel Diseases, Inflammatory)

\#2 TOPIC: (Urologic Neoplasms) OR TOPIC: (Urological Neoplasms) OR TOPIC: (Neoplasm, Urological) OR TOPIC: (Neoplasms, Urological) OR TOPIC: (Urological Neoplasm) OR TOPIC: (Urinary Tract Neoplasms) OR TOPIC: (Neoplasm, Urinary Tract) OR TOPIC: (Neoplasms, Urinary Tract) OR TOPIC: (Tract Neoplasm, Urinary) OR TOPIC: (Tract Neoplasms, Urinary) OR TOPIC: (Urinary Tract Neoplasm) OR TOPIC: (Neoplasms, Urologic) OR TOPIC: (Neoplasm, Urologic) OR TOPIC: (Urologic Neoplasm) OR TOPIC: (Cancer of Urinary Tract) OR TOPIC: (Urinary Tract Cancers) OR TOPIC: (Urological Cancer) OR TOPIC: (Cancer, Urological) OR TOPIC: (Cancers, Urological) OR TOPIC: (Urological Cancers) OR TOPIC: (Cancer of the Urinary Tract) OR TOPIC: (Urinary Tract Cancer) OR TOPIC: (Cancer, Urinary Tract) OR TOPIC: (Cancers, Urinary Tract) OR TOPIC: (Urologic Cancer)

\#3 \#1 and \#2 68 records 